\title{
The seroprevalence of Bartonella henselae in healthy adults in Korea
}

\author{
Hea Yoon Kwon ${ }^{1}$, Jae Hyoung Im¹, Sun Myoung Lee ${ }^{2}$, Ji Hyeon Baek ${ }^{1}$, Areum Durey ${ }^{1}$, Shin-Goo Park ${ }^{3}$, \\ Jae-Seung $\mathrm{Kang}^{4}$, and Jin-Soo Lee ${ }^{1}$
}

${ }^{1}$ Department of Internal Medicine, ${ }^{2}$ Clinical Research Center, Departments of ${ }^{3}$ Occupational Medicine and ${ }^{4}$ Microbiology, Inha University School of Medicine, Incheon, Korea

Received: December 29, 2015

Revised : March 24, 2016

Accepted: April 21, 2016

Correspondence to

Jin-Soo Lee, M.D

Department of Internal Medicine, Inha University School of Medicine, 27 Inhang-ro, Jung-gu, Incheon 22332, Korea

Tel: +82-32-890-2548

Fax: +82-32-890-2549

E-mail: ljinsoo@inha.ac.kr
Background/Aims: Cat-scratch disease (CSD), caused by Bartonella henselae is one of the most common zoonosis. However, only several cases of B. henselae infection have been reported in Korea. This study investigated the seroprevalence of $B$. henselae in healthy adults and related risk factors.

Methods: Serum samples from 300 healthy participants were analyzed using an immunoglobulin G immunofluorescence assay (IFA) for B. henselae isolated in Korea. Surveys on the risk factors for B. henselae infection were conducted simultaneously.

Results: Of the participants, $47.7 \%$ and $15.0 \%$ raised dogs and cats, respectively. The overall seroprevalence of B. henselae was 15.0\% (IFA titer $\geq 1: 64$ ). Participants who had raised cats showed $22.2 \%$ seropositivity against $B$. henselae, and those with no experience with cats showed $13.7 \%$ seroprevalence $(p=0.17)$. Participants who had cats as pets or been scratched by cats, showed $9.8 \%$ seropositivity against $B$. henselae (IFA titer $\geq 1: 256$ ). However, those who had not raised or been scratched by a cat showed $2.0 \%$ seropositivity $(p=0.015)$.

Conclusions: In Korea, the seroprevalence of $B$. henselae is higher than expected, suggesting that Bartonella infection due to B. henselae is not uncommon. Cats are proposed to play a more important role than dogs in transmission of CSD.

Keywords: Bartonella henselae; Seroepidemiologic studies; Korea; Cat-scratch disease

\section{INTRODUCTION}

Bartonella species have recently been proven to cause zoonosis. Bartonella spp. are gram-negative, intracellular bacterial pathogens, and 20 named species belong to the genus [1]. Among them, Bartonella henselae, Bartonella bacilliformis, and Bartonella quintana are important pathogens that cause infections in humans [1]. B. henselae is commonly transmitted when humans are scratched or bitten by cats or dogs [2-4]. Once infected, most people present with various clinical symptoms, including catscratch disease (CSD), trench fever, infective endocardi- tis, bacillary angiomatosis, lymphadenopathy, fever of infective endocarditis, fever of unknown origin, myalgia, neuropathy, and uveitis $[5,6]$.

In the United States, CSD caused by B. henselae is one of the most common zoonosis. The disease results in more than 25,000 patients per year [7]. Moreover, the seropositivity rates against $B$. henselae among healthy adults are $16.0 \%$ in Sweden, $8.7 \%$ in Spain, and 19.6\% in China [8-10].

In Korea, only a few cases of Bartonella infection have been reported [2]. A 14-year-old boy was reported as having cervical lymphadenopathy caused by Bartonella 
infection; this was the first proven case diagnosed with polymerase chain reaction (PCR) [11]. According to the Seoul Institute, 640,000 households (16.7\%) of Seoul citizens have pets [12]. Approximately, $16 \%$ to $43 \%$ of specimens (i.e., blood, nail, and saliva) from dogs and cats were positive for B. henselae, as confirmed by PCR [3]. Chae et al. [13] reported that $38.7 \%$ of patients with lymphadenopathy showed high titers of $B$. henselae immunoglobulin $\mathrm{G}(\mathrm{IgG})(\geq 1: 128)$.

These prior studies suggest that a higher incidence of B. henselae infection should be expected. The incidence of $B$. henselae infection and risk factors associated with CSD should be recognized in Korea, because many physicians are not aware of this disease. In this study, we investigated the seroprevalence of B. henselae in healthy Korean adults and related risk factors.

\section{METHODS}

\section{Study population}

Ethical approval was granted by the Institutional Review Board of Inha University Hospital (IUH-IRB 13-2793). Serum samples were obtained from study participants who visited Inha University Hospital in Incheon, Korea, for a health examination from January to December 2014. All participants provided informed consent.

\section{Questionnaire content}

The participants were asked to complete a questionnaire providing personal and epidemiological information on age, sex, living and working environment, history of contact with pets, experience of being scratched by pets, history of admission because of fever or lymphadenopathy, history of uveitis, level of physical activity, and history of travel abroad. All questionnaires were distributed by the two interviewers who were trained in advance regarding the interview contents.

\section{Serological testing and cultivation of Bartonella species}

$\mathrm{ECV}_{304}$ cells infected with a strain of $\mathrm{B}$. henselae, which was previously isolated from a clinical specimen (Genebank registration No.JQ638927.1) were grown and subjected to an in-house immunofluorescence assay (IFA) analysis [14]. The infected cells were smeared on the spot slides and fixed using acetone for 10 minutes at $4^{\circ} \mathrm{C}$. Sera from healthy participants were serially diluted from 1:40 to 1:1,280 and incubated with the pre-prepared slides at $37^{\circ} \mathrm{C}$ for 30 minutes. After washing three times with phosphate buffered saline, fluorescein-labeled goat anti-human IgG antisera was applied and the slides were incubated under the same conditions for the primary antibody reaction. Slides were mounted with anti-fluorescence media (Vector, Burlingame, CA, USA) and observed using a fluorescence microscope (Axioskop 2, CarlZeiss, Gottingen, Germany) at $\times 200$ magnification. The sera were simultaneously examined with commercially available slides for Bartonella-IFA IgG assay (Focus Diagnostics, Cypress, CA, USA), according to the inserts provided by the manufacturer.

The laboratory results based on the in-house IFA analysis were presented and two cutoff points for seropositivity were used: 1:64 and 1:256; 1:64 is the documented cut-off value, and 1:256 was used to include the proportion of participants who are more likely to have been infected or with presumptive evidence of recent infection with B. henselae.

\section{Statistical methods}

Data were analyzed using SPSS version 10.0 (SPSS Inc., Chicago, IL, USA). Univariate analysis (chi-square test and $t$ test) was performed to determine the impact of different independent risk factors.

\section{RESULTS}

Among 300 healthy participants, 113 (37.7\%) were men and 187 (62.3\%) were women. Sixty participants (20\%) were enrolled in each age group (i.e., 20 to 29,30 to 39,40 to 49,50 to 59 , and 60 years and older).

Corresponding questionnaires were completed by the 300 participants (Table 1). Of these, 143 (47.7\%) reported that they were raising or had raised dogs, 64 (15.0\%) had been scratched by dogs, 25 (8.3\%) were raising or had raised cats, and 39 (13.0\%) had tended animals other than dogs or cats. Additionally, five subjects (1.7\%) had been hospitalized because of febrile disease, and two patients had a history of lymphadenopathy. Forty-five of the participants $(15 \%)$ were seropositive for $B$. henselae IgG (1:64 or higher). In addition, among the seropositive 
Table 1. Questionnaire answers of the subjects $(n=300)$

\begin{tabular}{lc}
\hline Variable & No. of participants (\%) \\
\hline Experience of raising dogs & $143(47.7)$ \\
Experience of being scratched by dogs & $64(15.0)$ \\
Experience of raising cats & $25(8.3)$ \\
Experience of tending other animal & $39(13.0)$ \\
Hospitalization because of febrile illness & $5(1.7)$ \\
Experience of being scratched by other animals & $2(0.7)$ \\
\hline
\end{tabular}

Table 2. Age groups of the subjects with Bartonella henselae seropositivity of 1:64 or higher

\begin{tabular}{lccr}
\hline Age group, yr & Seronegative & Seropositive & Number \\
\hline $20-29$ & $55(91.6)$ & $5(8.3)$ & 60 \\
$30-39$ & $49(81.6)$ & $11(18.3)$ & 60 \\
$40-49$ & $54(90.0)$ & $6(10.0)$ & 60 \\
$50-59$ & $50(83.3)$ & $10(16.6)$ & 60 \\
$>60$ & $47(78.3)$ & $13(21.6)$ & 60 \\
Total & 255 & 45 & 300 \\
\hline
\end{tabular}

Values are presented as number (\%).

individuals, the proportion of men (51.1\%) was slightly higher than that of women (48.9\%); however, the difference was not statistically significant $(p=0.44)$. Each age group showed varying seropositivity, ranging from $8.3 \%$ to $21.7 \% ; 8.3 \%$ for the 20 to 29 age-group, $18.3 \%$ for the 30 to $39,10.0 \%$ for the 40 to $49,16.6 \%$ for the 50 to 59 , and $21.6 \%$ for the group aged 60 years or above. Individuals aged older than 60 years had the highest rate of seroprevalence, but this difference was not statistically significant $(p=0.24)$ (Table 2, Fig. 1).

The seroprevalence of $B$. henselae (IgG titer of $1: 64$ or higher) in this study was $15.0 \%$ (45/300) (Table 3). The individuals who were $B$. henselae seropositive (IgG titer of 1:64) were more likely to have raised or been scratched by cats. However, the results were not statistically significant.

Ten of the participants (3.3\%) had B. henselae IgG titers of 1:256 or higher (Table 4); five (3.5\%) were among the 143 individuals who had raised dogs and five (3.2\%) were among the 157 participants who had not raised dogs. B. henselae seroprevalence of $8.9 \%$ (4/45) was observed among the participants who had raised cats and $2.4 \%(6 / 255)$ among those who had a history of raising cats, and this result was statistically significant $(p=0.05)$. Among those who had a history of raising cats and be-

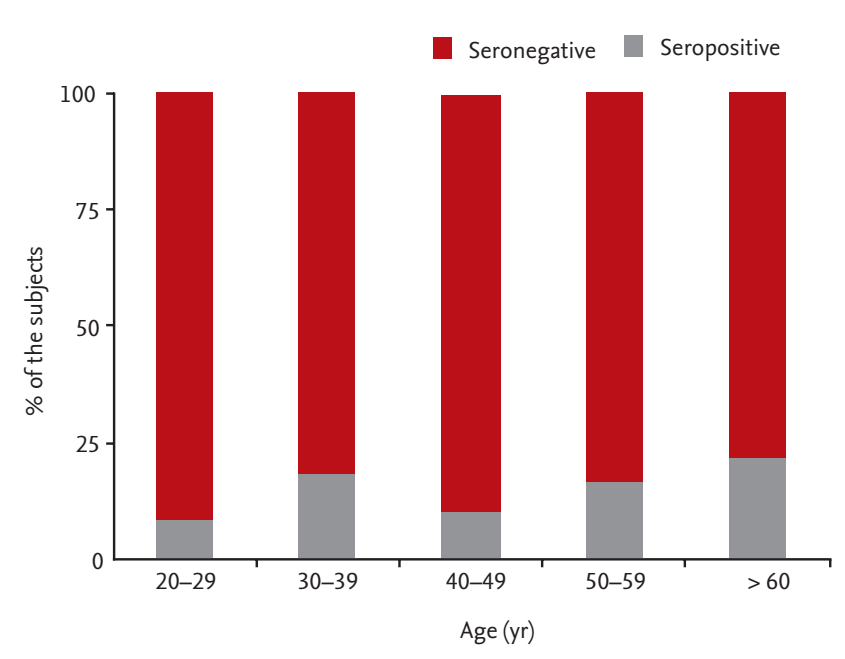

Figure 1. Graph by age grouping of the participants with Bartonella henselae immunoglobulin G titers of 1:64 or higher.

ing scratched by cats, 9.8\% (5/51) were seropositive for B. henselae. Among those who did not have a history of raising cats and having been scratched by cats, 2.0\% (5/249) were seropositive, and this result was statistically significant $(p=0.02)$.

However, a history of raising dogs or a history of raising and being scratched by dogs was not significantly associated with higher titers (i.e., 1:256 or higher) $(p=0.57$ 
Table 3. Bartonella henselae seroprevalence of 1:64 or higher according to risk factors

\begin{tabular}{|c|c|c|c|}
\hline \multirow{2}{*}{ Risk factor } & \multicolumn{2}{|c|}{ Seropositive subject } & \multirow{2}{*}{$p$ value } \\
\hline & With risk factor & Without risk factor & \\
\hline Have raised dogs & $23 / 143(16.1)$ & $22 / 157(14.0)$ & 0.63 \\
\hline Have been scratched by dogs & $9 / 64(14.1)$ & $36 / 236(15 \cdot 3)$ & 1.00 \\
\hline Have raised cats & $10 / 45(22.2)$ & $35 / 255(13 \cdot 7)$ & 0.17 \\
\hline Have been scratched by cats & $5 / 25(20.0)$ & $40 / 275(14.5)$ & 0.31 \\
\hline Have raised dogs and have been scratched by dogs & $25 / 160(15.6)$ & $20 / 140(14 \cdot 3)$ & 0.87 \\
\hline Have raised cats and have been scratched by cats & $11 / 51(21.6)$ & $34 / 249(13.7)$ & 0.19 \\
\hline Have raised dogs and cats & $25 / 148(16.9)$ & $20 / 152(13.2)$ & 0.42 \\
\hline Have raised and have been scratched by dogs or cats & $27 / 168(16.1)$ & $18 / 132(13.6)$ & 0.63 \\
\hline
\end{tabular}

Values are presented as number (\%).

Table 4. Bartonella henselae seroprevalence of 1:256 or higher according to the risk factors

\begin{tabular}{|c|c|c|c|}
\hline \multirow{2}{*}{ Risk factor } & \multicolumn{2}{|c|}{ Seropositive subject } & \multirow{2}{*}{$p$ value } \\
\hline & With risk factor & Without risk factor & \\
\hline Have raised dogs & $5 / 143(3 \cdot 5)$ & $5 / 157(3.2)$ & 0.57 \\
\hline Have been scratched by dogs & $0 / 64(0.0)$ & $10 / 236(4.2)$ & 0.09 \\
\hline Have raised cats & $4 / 45(8.9)$ & $6 / 255(2.4)$ & $0.05^{\mathrm{a}}$ \\
\hline Have been scratched by cats & $2 / 25(8.0)$ & $8 / 275(2.9)$ & 0.20 \\
\hline Have raised dogs and have been scratched by dogs & $5 / 160(3.1)$ & $5 / 140(3.6)$ & 0.54 \\
\hline Have raised cats and have been scratched by cats & $5 / 51(9.8)$ & $5 / 249(2.0)$ & $0.02^{\mathrm{a}}$ \\
\hline Have raised dogs and cats & $7 / 148(4.7)$ & $3 / 152(2.0)$ & 0.16 \\
\hline Have raised and have been scratched by dogs or cats & $6 / 168(4.2)$ & $3 / 132(2.3)$ & 0.28 \\
\hline
\end{tabular}

Values are presented as number (\%).

${ }^{a}$ Statistically significant result.

and $p=0.54$, respectively).

In this experiment, both in-house and commercial IFA slides were used to detect B. henselae seropositivity. B. henselae isolated from Korea was used as the antigen for in-house slides to improve the accuracy of the detection. The sensitivity of assays using in-house slides compared those using commercial slides was $84.8 \%$, and the specificity was $97.6 \%$, suggesting that the in-house assays are reliable for assessment of $B$. henselae antibody titer (Table 5).

\section{DISCUSSION}

Several seroepidemiologic studies in healthy populations have been conducted to investigate Bartonella spp. seroprevalence. The seroprevalence of Bartonella infection varies by region. A study of blood donors in Swe- den showed $16.1 \%$ seropositivity for B. henselae and B. quintana [8]. Seroprevalence of B. henselae among healthy adults was reported to be $8.7 \%$ in Spain and $19.6 \%$ in Zhejiang province in China [9,10].

Only a few cases of $B$. henselae infection have been reported in Korea. However, B. henselae DNA has been detected in specimens from the reservoir of animals such as cats and dogs, and higher titers for B. henselae were documented in patients with lymphadenopathy [3]. These prior studies indicate a high number of unreported patients with Bartonella infection. In the current study, $15.0 \%$ of asymptomatic healthy Korean adults were seropositive for B. henselae (IgG, 1:64 or higher). In addition, 3.0\% of healthy participants had IgG titers of 1:256 or higher, indicating the greater likelihood of a recent infection. Bartonella-IgG IFA is known to have cross reactivity with other diseases $[15,16]$. Even considering that, however, $15.0 \%$ seroprevalence suggests that Bar- 
Table 5. Sensitivity and specificity of IFA results: commercial vs. in-house

\begin{tabular}{lcr}
\hline \multirow{2}{*}{ Variable } & \multicolumn{2}{c}{ In house IFA } \\
\cline { 2 - 3 } Commercial IFA & Positive & Negative \\
Positive & $39(84.8)$ & $7(15.2)$ \\
Negative & $6(2.4)$ & $248(97.6)$ \\
\hline
\end{tabular}

Values are presented as number (\%).

IFA, immunofluorescence assay.

tonella infection is not uncommon in Korea.

B. henselae infection may present with various nonspecific clinical symptoms, which causes difficulty in the diagnosis of this infection. Also, in pathological examination, the infection demonstrates granulomatous lymphadenitis. Therefore, it may be misdiagnosed as tuberculosis or Kikuchi-Fujimoto disease, which is known to be more common causes of lymphadenitis in Korea [17]. Furthermore, since B. henselae is sensitive to antibiotics such as rifampicin, it may improve clinically with the anti-tuberculosis medication. In Korea, the high incidence of Kikuchi disease has been reported as a cause of lymphadenopathy. Physicians should be aware that $B$. henselae infection is also an important cause of lymphadenopathy.

B. henselae infection is a zoonosis, and animals play an important role in its transmission. Cats are known to be the most important transmitter, although other animals including dogs may play a role as well [3]. In a study conducted in Spain, 31.6\% of seropositive individuals reported exposure to cats and 5.6\% of people reported exposure to dogs [9]. In our study, 143 participants (47.7\%) had experience tending dogs, while only 25 (8.3\%) had tended cats. Traditionally, Koreans have had a predilection for dogs above cats. Therefore, we expected that dogs may serve as a reservoir for B. henselae infection. Chae et al. [13] did not provide evidence of a statistically significant association of $B$. henselae seropositivity with raising animals, including cats. In our study, tending cats or a previous experience of tending cats showed a statistically significant association with $B$. henselae seropositivity. The experience of tending dogs did not show a statistically significant association with $B$. henselae seropositivity. Therefore, our study concludes that cats are more strongly associated with $B$. henselae infection than dogs, which corresponds with previous studies.

Our study showed that the seroprevalence of B. henselae among healthy Korean adults is also high and seropositivity is associated with history of tending cats or being scratched by cats. Physicians should be aware of CSD, considering the rate of $B$. henselae seropositivity among the healthy Korean adults in this study was 15.0 $\%$. In addition, further study is needed to determine whether Bartonella infection causes clinical symptoms such as lymphadenitis, acute febrile illness, and uveitis in adults.

\section{KEY MESSAGE}

1. In Korea, Bartonella infection is neglected but common disease. Physicians need to have high suspicion to diagnose the disease.

2. Contact with cats (raising cats, scratch) may play an important role in transmission of Bartonella henselae.

\section{Conflict of interest}

No potential conflict of interest relevant to this article was reported.

\section{REFERENCES}

1. Mandell GL, Douglas RG, Bennett JE, Dolin R, Blaser MJ. Mandell, Douglas, and Bennett's Principles and Practice of Infectious Diseases. 8th ed. Vol. 2. Philadelphia: Elsevier, 2015 .

2. Kim MH, Kim BN, Han TH. Cat-scratch disease: a case report and literature review of human and animal studies performed in Korea. Infect Chemother 2012;44:299-302.

3. Kim YS, Seo KW, Lee JH, et al. Prevalence of Bartonella henselae and Bartonella clarridgeiae in cats and dogs in Korea. J Vet Sci 2009;10:85-87.

4. Sun J, Fu G, Lin J, Song X, Lu L, Liu Q. Seroprevalence of Bartonella in Eastern China and analysis of risk factors. BMC Infect Dis 2010;10:121.

5. Anderson BE, Neuman MA. Bartonella spp. as emerging human pathogens. Clin Microbiol Rev 1997;10:203-219.

6. Florin TA, Zaoutis TE, Zaoutis LB. Beyond cat scratch disease: widening spectrum of Bartonella henselae infec- 
tion. Pediatrics 2008;121:e1413-e1425.

7. Jackson LA, Perkins BA, Wenger JD. Cat scratch disease in the United States: an analysis of three national databases. Am J Public Health 1993;83:1707-1711.

8. McGill S, Wesslen L, Hjelm E, et al. Bartonella spp. seroprevalence in healthy Swedish blood donors. Scand J Infect Dis 2005;37:723-730.

9. Pons I, Sanfeliu I, Cardenosa N, Nogueras MM, Font B, Segura F. Serological evidence of Bartonella henselae infection in healthy people in Catalonia, Spain. Epidemiol Infect 2008;136:1712-1716.

10. Liu Q, Eremeeva ME, Li D. Bartonella and Bartonella infections in China: from the clinic to the laboratory. Comp Immunol Microbiol Infect Dis 2012;35:93-102.

11. Sander A, Posselt M, Oberle K, Bredt W. Seroprevalence of antibodies to Bartonella henselae in patients with cat scratch disease and in healthy controls: evaluation and comparison of two commercial serological tests. Clin Diagn Lab Immunol 1998;5:486-490.

12. Chung JY, Koo JW, Kim SW, Yoo YS, Han TH, Lim SJ. A case of cat scratch disease confirmed by polymerase chain reaction for Bartonella henselae DNA. Korean J Pediatr 2005;48:789-792.

13. Chae MB, Lee JY, Kwak YG, et al. Prevalence of antibodies to Bartonella henselae and Bartonella quintana in Korean patients with lymphadenopathy. Korean J Infect Dis 2002;34:305-310.

14. Yoo K, Cho S, Gin Y, Lee Y. Strategic Guidelines to Protect and Manage Pet Animals in Seoul. Seoul: Seoul Development Institute, 2004.

15. Kil SH, Kang JS. Production of the monoclonal antibodies against Bartonella henselae isolated from a Korean patient. J Bacteriol Virol 2012;42:41-47.

16. La Scola B, Raoult D. Serological cross-reactions between Bartonella quintana, Bartonella henselae, and Coxiella burnetii. J Clin Microbiol 1996;34:2270-2274.

17. Maurin M, Eb F, Etienne J, Raoult D. Serological cross-reactions between Bartonella and Chlamydia species: implications for diagnosis. J Clin Microbiol 1997;35:22832287. 